\title{
Cognitive Development in malnourished and stunted children
}

Setyo Handryastuti, Hardiono D. Pusponegoro, Ivan Riyanto Widjaja, Achmad Rafli Department of Child Health, Faculty of Medicine, University of Indonesia

Cipto Mangunkusumo Hospital, Jakarta

\section{Background}

In Indonesia, prevalence of stunting is $30.8 \%$ while malnutrition is $17.7 \%$. Stunting and malnutrition might be related to impaired cognitive development.

\section{Aims}

To determine cognitive development in stunted children, malnourished children without stunting and constitutional short stature.

\section{Methods}

Cross sectional study was done in Cipto Mangunkusumo Hospital Pediatric Clinic to children aged 6 month-3 years old. Anthropometry was calculated based on WHO growth chart. Cognitive development was assessed with Bayley Scales of Infant Development Third Editions (BAYLEY-III).

\section{Results}

Fifty-eight children aged less than 3 years were enrolled in this study (26 children with stunting, 25 children with malnutrition without stunting, and 7 children with constitutional short stature). Age of subjects were 11(2.0-34.0), 13.0(2.0-38.0), and 26.0(16.0-33.0) respectively. Bayley-III percentile in cognitive scale were 12.5(0.1-75), 16(0.1-99.9), 37.0(0.1-99) $(p=0.274)$

\section{Discussion}

Stunting children showed worst cognitive function compared to malnourished children and constitutional short stature, while malnourished children were worse compared to constitutional short children, although it was not significant.

Difference in age and small number of subjects in constitutional short stature was related to difficulty in diagnosing constitutional short stature in children aged $<3$ years.

\begin{tabular}{|c|c|c|c|c|}
\hline & $\begin{array}{c}\text { Stunting } \\
(n=26)\end{array}$ & $\begin{array}{l}\text { Malnutriti } \\
\text { on } \\
\text { without } \\
\text { stunting } \\
(n=25)\end{array}$ & $\begin{array}{c}\text { Constitutio } \\
\text { nal short } \\
\text { stature } \\
(n=7)\end{array}$ & $\mathbf{p}$ \\
\hline $\operatorname{Sex}(n)$ & & & & - \\
\hline Male & 16 & 14 & 4 & \\
\hline Female & 10 & 11 & 3 & \\
\hline Age (month) & $\begin{array}{c}11 \\
(2-34)\end{array}$ & $16 \pm 9.63$ & $26 \pm 5.16$ & 0.02 \\
\hline
\end{tabular}

\section{BAYLEY-III percentile}

\begin{tabular}{|c|c|c|c|c|}
\hline & $\begin{array}{l}\text { Stunting } \\
(n=26)\end{array}$ & $\begin{array}{c}\text { Malnutriti } \\
\text { on } \\
\text { without } \\
\text { stunting } \\
(n=25)\end{array}$ & $\begin{array}{c}\text { Constitutio } \\
\text { nal short } \\
\text { stature } \\
(n=7)\end{array}$ & $\mathbf{p}$ \\
\hline \multicolumn{5}{|l|}{ Percentile } \\
\hline Cognitive & $\begin{array}{c}12.5 \\
(0.1-75)\end{array}$ & $\begin{array}{c}16 \\
(0.1-99.9)\end{array}$ & $44.7 \pm 36.82$ & 0.274 \\
\hline Language & $\begin{array}{c}20.5 \\
(0.1-95)\end{array}$ & $\begin{array}{c}18 \\
(0.2-94)\end{array}$ & $37.1 \pm 33.37$ & 0.892 \\
\hline Motor & $\begin{array}{c}1 \\
(0.1-75)\end{array}$ & $\begin{array}{c}4 \\
(0-79)\end{array}$ & $\begin{array}{c}16 \\
(0.1-75)\end{array}$ & 0.109 \\
\hline $\begin{array}{l}\text { Social- } \\
\text { emotional }\end{array}$ & $\begin{array}{c}25 \\
(0.4-99)\end{array}$ & $\begin{array}{c}16 \\
(1-99.9)\end{array}$ & $\begin{array}{c}16 \\
(1-98)\end{array}$ & 0.779 \\
\hline $\begin{array}{l}\text { Adaptive } \\
\text { behavior }\end{array}$ & $\begin{array}{c}7 \\
(0.1-75) \\
\end{array}$ & $\begin{array}{c}12 \\
(0.1-58) \\
\end{array}$ & $11.6 \pm 12.63$ & 0.780 \\
\hline
\end{tabular}

\section{Cognitive percentile}

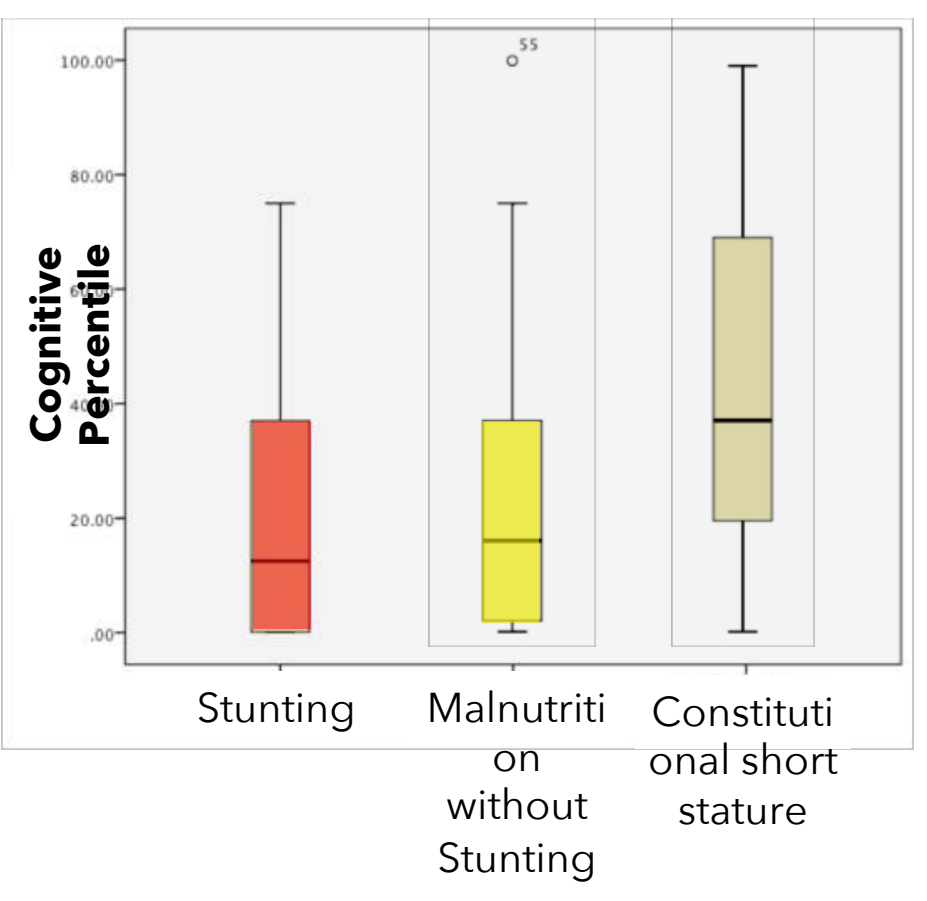

Language percentile

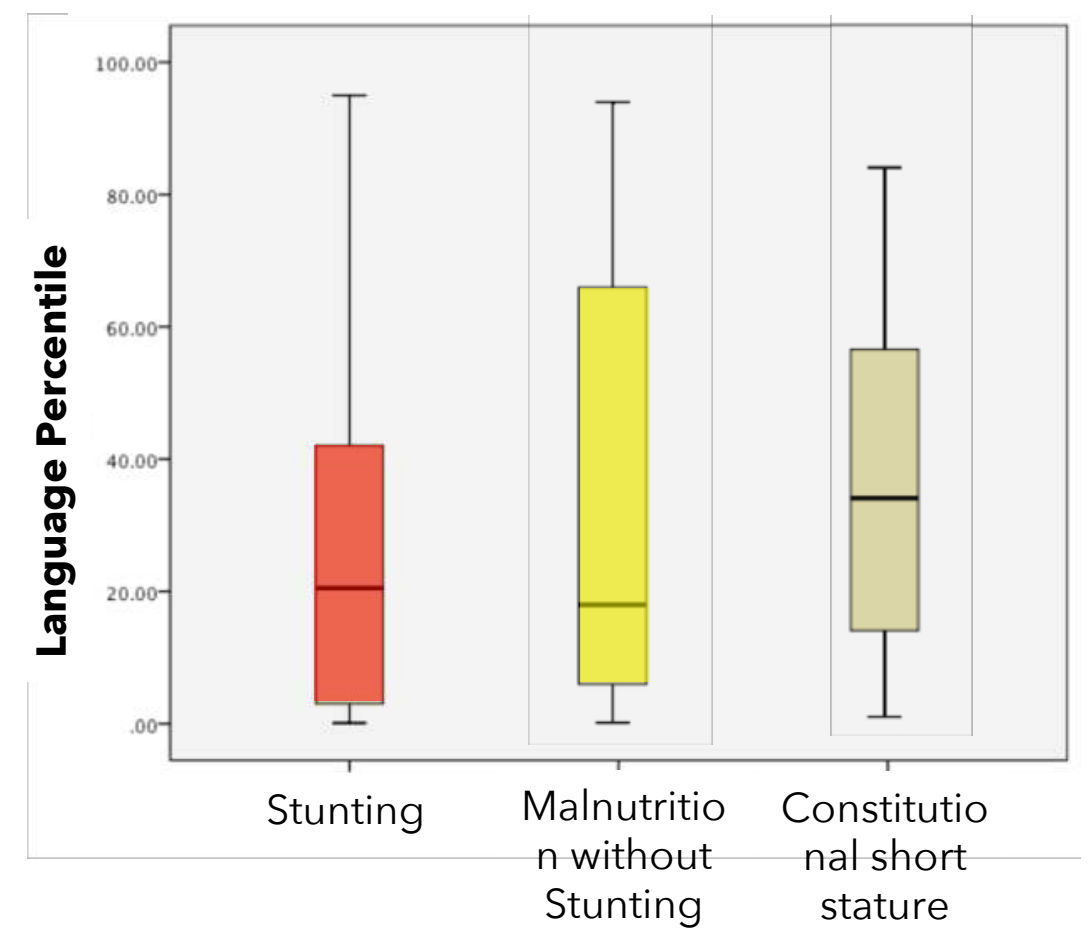

Motor percentile

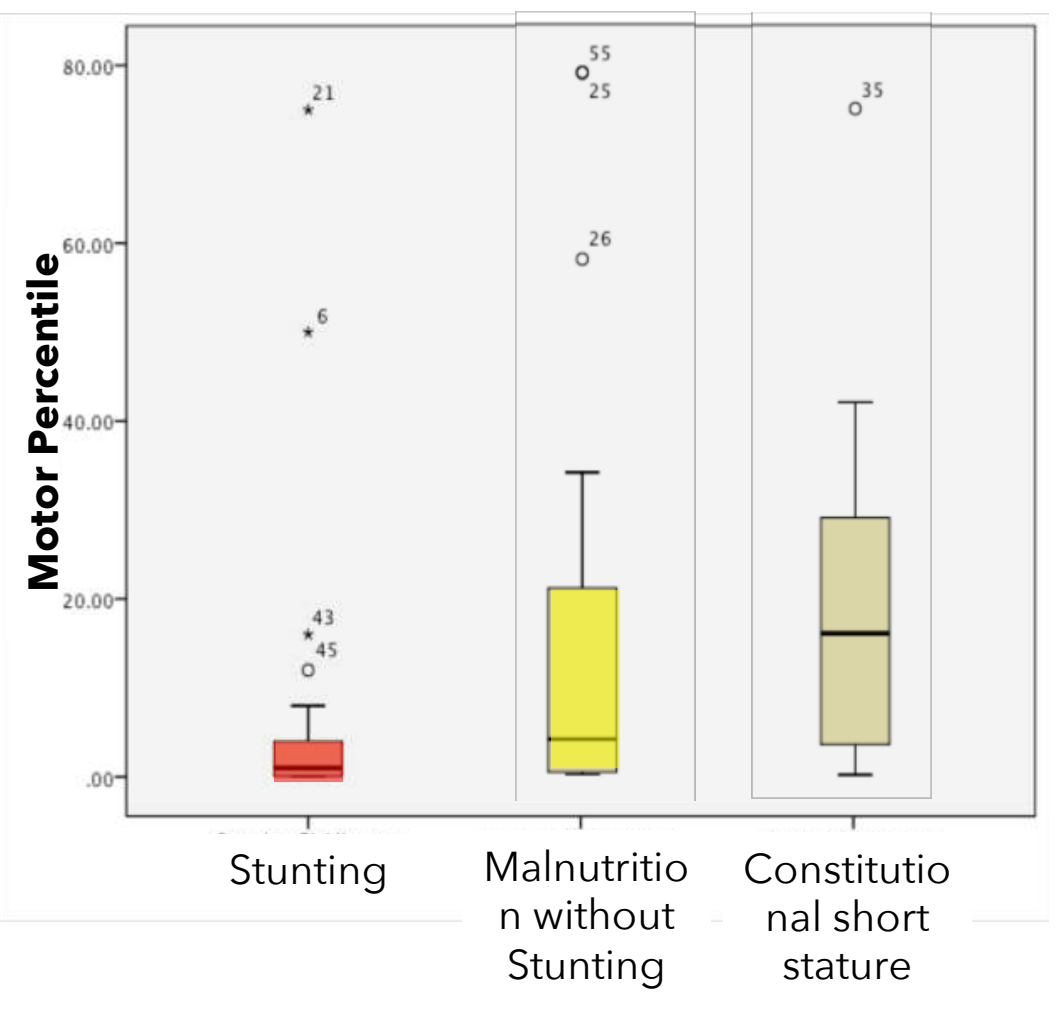

